\title{
Development of Heating Device for Drawer Type Circuit Breaker
}

\author{
Lei Zhang, Bo Fang, Pin Dong, Tianci Li, Jia Liu, Rui Tong, \\ Xiao Liu, Jingyi Zhang, Yanjun Pang \\ Fushun Power Supply Company, Liaoning Electric Power Company Limited, State Grid, China
}

\begin{abstract}
Keywords: Drawer Type; Heating Device; Circuit Breaker; Silicon Rubber Electrothermal Film; Field Application
\end{abstract}

\begin{abstract}
High voltage circuit breaker is the most important control and protection equipment in power system. Northern winter weather is cold, and the breaker mechanism box relies on the heating device to raise the temperature, thus ensure the normal operation of the circuit breaker. Now the heating plate of the $100 \mathrm{~W}$ aluminum alloy is adopted, it is easy to damage, therefore, how to improve the electric heating plate has become an urgent problem to be solved. Under the premise of the original appearance, the internal heat radiating element is changed into a drawer type electrothermal film module of silicon rubber. The outer package of the module is provided with a concave aluminum envelope, its bottom is equipped with a pilot lamp for working status indicator, this module can be used as long as it is inserted into the groove of the original heating plate, the service life is long, and the replacement is convenient.
\end{abstract}

\section{Introduction}

High voltage circuit breaker is the most important control and protection equipment in power system. Control action is according to the power grid operation need, can safely and reliably input or withdraw from the corresponding line or electrical equipment, safety action is in the line or electrical equipment failure, can quickly remove the fault part from the power grid, to protect the normal operation of the power grid fault free part. High voltage transmission and distribution lines require high voltage circuit breakers with automatic switching function, to ensure the normal operation of the power grid. In short, it is required to be able to reliably switch to the circuit in accordance with the needs of normal or accident. The functions of high voltage circuit breakers are as follows:

(1) It should be a good conductor in the closed state, not only to the normal current should be able to withstand its thermal and mechanical action, but also to the short-circuit current should be so.

(2) It has good insulating properties between the ground, the interphase and the fracture surface.

(3)At any point in the closed state, it should be able to open the current below the rated current in a short period of time without the risk of the occurrence of the voltage.

(4) At any point in the open state, it should be able to close the current in the short circuit safely without the contact melting welding ${ }^{[1]}$.

\section{The Components of the Circuit Breaker}

\section{Conductive part.}

The conductive part is the path of the current, is usually made of conductive rod, isolator gate, beam, flexible connection , contact terminal etc. The contact is the most important part of the conductive part, the circuit breaker is to rely on him to connect or disconnect the current.

Due to operation of the circuit breakers in the long run pass through the over load current, the conductive loop generates heat, when the contact resistance is large contact, heating will be more powerful, even burn out.

\section{Arc extinguishing device.}

The arc extinguishing device of the circuit breaker is the heart of the circuit breaker. Because the contact can only cut off the current, and the arc extinguishing device can extinguish the arc which is 
generated by the contact in the off current. Therefore, its good and bad determines the breaking capacity of circuit breakers to a great extent.

\section{Insulation system.}

The following three aspects must be guaranteed in the circuit breaker.

(1) The insulation between the charged part and the ground part, mainly the porcelain bushing, the lifting rod, etc.

(2) When the circuit breaker is in the open position, the insulation between the fracture surface is usually guaranteed by insulation oil (vacuum or SF6)

(3) The insulation of the phase, three-phase oil circuit breaker mainly rely on insulation oil, insulation separator to insulation.

\section{Operating mechanism.}

The operation mechanism is an important part of the circuit breaker, it can ensure that the contact system in a certain way and a certain speed when divide-shut brake, reliable connection and disconnection of the circuit, The operation of mechanical oil is manual, electromagnetic, spring type. Require him to move flexible and reliable. It requires action flexible and reliable ${ }^{[1]}$.

\section{Heating device.}

A heating device is arranged in a circuit breaker mechanism box, it can raise the temperature in the box to ensure the normal operation of the circuit breaker.

\section{The Shortage of Heating Device}

Northern winter weather is cold, the breaker mechanism box needs to rely on the heating device to raise the temperature in the chamber to ensure the normal operation of the circuit breaker. 100W aluminum alloy plate heater is used in all circuit breakers in Fushun area, The type heating plate adopts a resistance wire inside, the $220 \mathrm{~V}$ voltage is set up on both ends to cause the resistance wire to heat and it increases the temperature in the mechanism box, but the heat could not be distributed which leads to the resistance wire breakage, damage to the heating system.

\section{The Design Principle of the Drawer Type Circuit Breaker Heating Device}

Aiming at the problems in the existing technology, based on the excellent properties of silicone rubber electric heating module, and through the heating effect comparison, the article provides a drawer type heating device for $66 \mathrm{kV}$ and the above breaker mechanism, which solves the problem of the damage of the heating device in the prior art.

The design scheme is as follows:

Under the premise of the original appearance, the inner heat radiating element is replaced into a drawer type silicon rubber electrothermal film module, This module uses silicone rubber which has high temperature $\left(-60^{\circ} \mathrm{C}\right.$ to $\left.250^{\circ} \mathrm{C}\right)$, high thermal conductivity (thermal conductivity coefficient of 0.83), good insulation performance ,good strength, high temperature resistant fiber reinforced material and soft electric heating membrane element which is composed of nickel chromium alloy resistance wire or circuit with metal foil heating film, the outer package of the module is provided with a concave aluminum envelope, its bottom is equipped with a pilot lamp for working status indicator, drawer type silicon rubber electrothermal module is inserted into the groove of the original heating plate. 


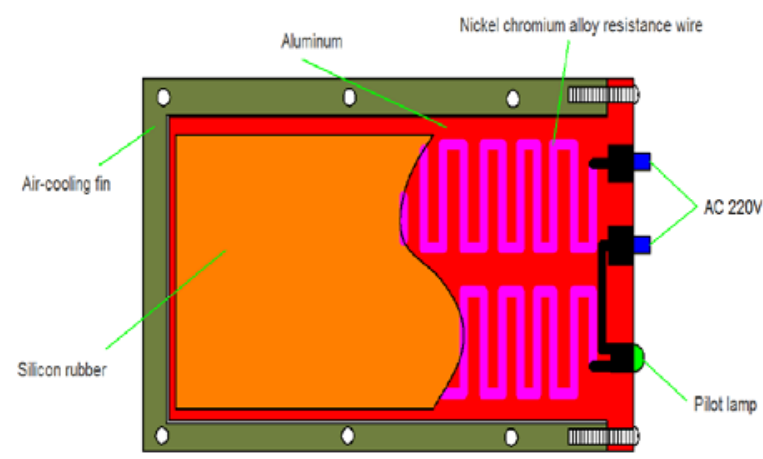

Fig.1. Cutaway view of the silicon rubber electrothermal module

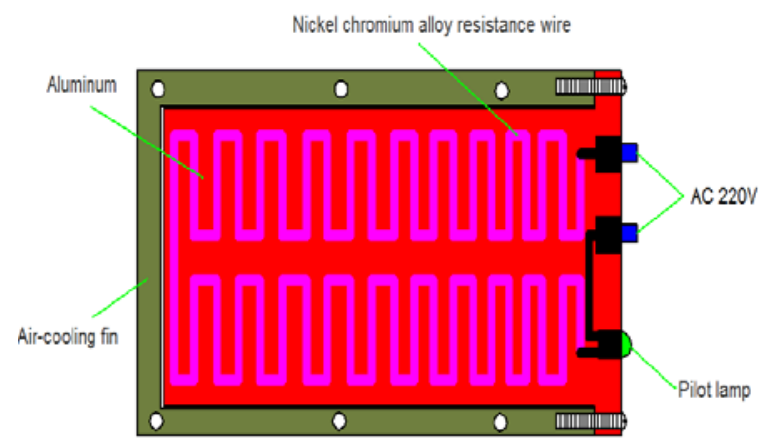

Fig.2. Internal structure diagram of the silicon rubber electrothermal module

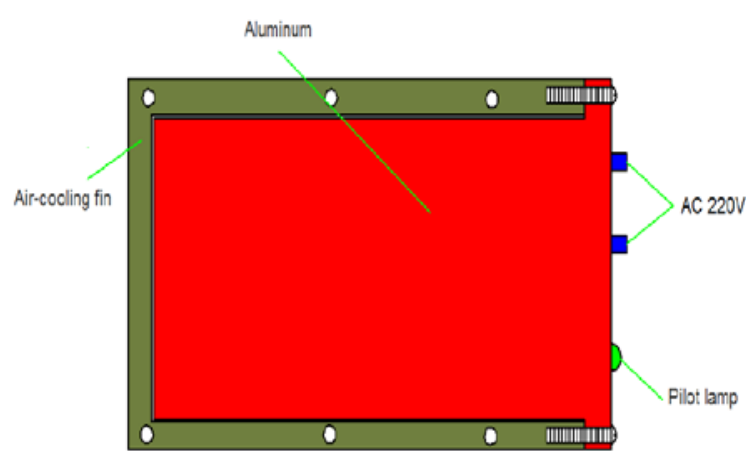

Fig.3. Installation diagram the silicon rubber electrothermal module

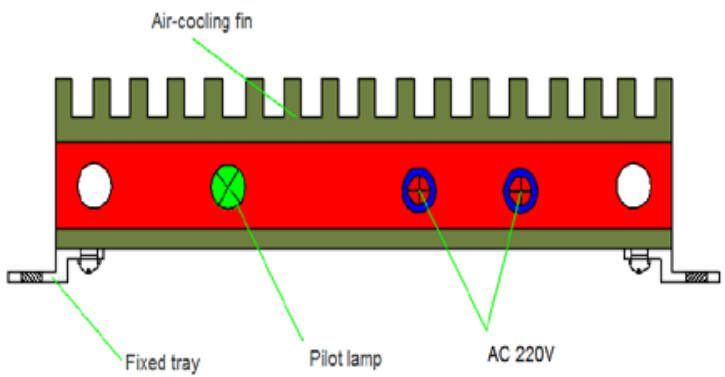

Fig.4. Bottom sketch map after installed

\section{Field Application}

The drawer type silicon rubber heating module is installed in the $220 \mathrm{kV}$ bus circuit breaker mechanism box of the ShengLi 220kV substation, since the installation in November 30, 2015, the circuit breaker mechanism box is tested and controlled by a thermometer at $20{ }^{\circ} \mathrm{C}$, and no hysteresis circuit breaker occurred. 
The drawer type silicon rubber heating module is installed in the 66kV PingLvYi line circuit breaker mechanism box of the HePing $220 \mathrm{kV}$ substation, since the installation in November 30 , 2015, the circuit breaker mechanism box is tested and controlled by a thermometer at $20{ }^{\circ} \mathrm{C}$, and no hysteresis circuit breaker occurred.

The advantages of the device are as follows: The silicon rubber electrothermal module has excellent physical strength and flexibility, and can make the electric heating element and the heated object in good contact; It can be designed into various shapes; it has light weight, thin thickness, small heat capacity, and can achieve very fast heating rate; The precision metal electrothermal circuit can further improve the surface power density of the silicon rubber heating element, improve the uniformity of the surface heating power, prolong the service life and have a good control performance; Silicone rubber has good weather resistance and aging resistance, as the surface insulation material of electric heating film, it can effectively prevent the product surface crack and enhance the mechanical strength, and greatly extend the service life of the product; Silicon rubber electrothermal module has good resistance to chemical corrosion resistance, used for wet, corrosive gas and other places of relatively poor environment; It is easy to install; the pilot lamp indicates the working status correctly, prevents the accident of scald when the heating plate is checked.

\section{Conclusions}

The design and manufacture of the drawer type heating device is simple, the application is also very convenient, and solve practical problems, so it can be applied to the field work. Through the practical work, we can know that our overall design ideas are correct and clear. Drawer type heating device successfully solved the problem that the circuit breaker mechanism box is not easy to replace the internal heating device because of the cold winter in northern, thereby ensuring the normal operation of the circuit breaker mechanism. Moreover, the bottom of this device is equipped with a pilot lamp for working status indicator, this module can be used as long as it is inserted into the groove of the original heating plate, the service life is long, and the replacement is convenient, so it has a wide range of promotional value and application prospects.

\section{References}

[1] Dan Wenpei, Wang Bing, Qi Ling. An Eample of Eectrical Euipment Testing and Fault Treatment. China Water Conservancy and Hydropower Press, 2012.6

[2] Chen Huagang. Preventive Test Method and Diagnosis Technology of Electric Power Equipment [M]. Beijing science and Technology Press.2001

[3] Yang Guangyuan. Analysis of The cause of Circuit Rresistance of LW24-72.5Type Circuit Breaker in Guangxi Electric Power.2011 